\title{
Monitoramento do espelho d'água da represa do Vossoroca entre 2010-2015 usando dados Landsat
}

\author{
Water surface monitoring at the Vossoroca Dam between 2010-2015 using Landsat data
}

\author{
Gislaine do Rocio Mello Pinto ${ }^{1}$ \\ Jorge Antonio da Silva Centeno ${ }^{2}$ \\ ${ }^{1}$ Pós-Graduação em Geoprocessamento - Pontifícia Universidade Católica do Paraná - Rua Imaculada Conceição, \\ 1155 Bairro Prado Velho Cep 80215-90. Curitiba, PR, Brasil. \\ gislainermp@ hotmail.com \\ ${ }^{2}$ Universidade Federal do Paraná - UFPR Caixa Postal 19001, Cep 81531-990. Curitiba, PR, Brasil. \\ centeno@ufpr.br
}

Recebido em 02/03/2016 - Aceito em 09/12/2016

Received on 02/03/2016 - Accepted on 09/12/2016

\begin{abstract}
RESUMO - O presente artigo analisa e discute a variação do espelho d'água da Represa do Vossoroca, referente ao verão dos anos de 2010, 2012, 2014 e 2015. A variação temporal do espelho d'água do reservatório do Vossoroca é analisada com base em imagens de satélite LANDSAT, visando identificar as principais alterações no espelho d'água. A análise compreendeu o georreferenciamento de imagens, delimitação de poligonais, estatísticas de área com o cruzamento e avanço da lâmina d'água. Dois métodos são comparados: o primeiro baseado na classificação pela máxima verossimilhança Gaussiana e o segundo usando o segmentador Mean Shift. Utilizando 2010 como referência principal, estabeleceu-se o comparativo com os anos de 2012, 2014 e 2015. Notou-se a forte redução do espelho de água em 2014 e provou-se que o método baseado na segmentação proporciona estimativas próximas daquelas obtidas pelo método estatístico.
\end{abstract}

Palavras-chave: espelho de água; sensoriamento remoto; segmentação; recursos hídricos.

ABSTRACT - In the present paper it is analyzed and discussed the use of remote sensing imagery to monitor the variation of the water surface of the Vossoroca dam, referring to the summer of the years 2010, 2012,2014 and 2015. The temporal variation of the water surface of the reservoir is analyzed using Landsat satellite imagery to identify the main changes in its surface area. The analysis included the georeferencing of images, delineation of polygonal area statistics with the intersection and advancement of water depth. Two methods are compared: the first based on the Gaussian maximum likelihood classification and the second using the Mean Shift segmention.Using 2010 as main reference, established the comparison with the years 2012, 2014 and 2015, referring to the mentioned periods. It was noted the strong reduction of the water mirror in 2014 and it was proved that the method based on segmentation provides estimates close to those obtained by statistical method.

Keywords: water surface variation; remote sensing; segmentation; water resources.

\section{INTRODUÇÃO}

No sul do Brasil o inverno normalmente caracterizase por períodos mais secos e o verão por meses chuvosos, observando-se a variação sazonal de precipitação (Nery e Silva, 2001). Outras variações com menor freqüência caracterizam também anos mais secos ou mais chuvosos.

Longos períodos secos afetam a vida e a economia na região, a geração de energia, a agricultura e a pecuária. A atividade econômica o abastecimento urbano e a geração de energia elétrica podem sofrer fortemente os efeitos de períodos de seca, quando a estiagem reduz a disponibilidade hídrica, associada ao volume de água armazenado em reservatórios.
A barragem do Vossoroca perto de Curitiba é um exemplo de usina de geração elétrica que depende da precipitação. Nos anos entre 2010 e 2015 a região sofreu com um longo período de secas, que afetou a reserva hídrica no sul e sudoeste do Brasil.

No presente artigo é abordado o problema do monitoramento da reserva hídrica, através do acompanhamento do espelho de água dos reservatórios, analisando principalmente a situação crítica sofrida nos anos 2010 a 2015.

O artigo é organizado da seguinte forma. Inicialmente são apresentados aspectos teóricos que fundamentam a pesquisa, focalizando a influência da precipitação no espelho d'água na represa do Vossoroca e a importância da utilização das análises de imagens 
Landsat. A seguir são descritos os métodos usados na pesquisa e finalmente são apresentados os resultados e a discussão do estudo.

\section{REVISÃO DE LITERATURA}

Devido à escala do objeto de estudo, o uso de sensoriamento remoto foi estudado para o delineamento de reservatórios desde o lançamento do Landsat MSS, como pode ser lido em Smith (1997). Com o desenvolvimento de novos sensores e melhoras nas resoluções das imagens, estes métodos forneceram melhores resultados e estudos científicos comprovam a viabilidade do uso de imagens orbitais. Alguns exemplos são citados a seguir.

Um dos principais estudos é aquele realizado por Frazier and Page (2000), o que analisaram quantitativamente a precisão do delineamento de espelhos de água em dados Landsat TM. O estudo revelou que pode- se atingir precisão de até $96,9 \%$ aplicando a limiarização à banda 5 do sensor TM (Infra-vermelho médio) e que esta técnica se equivale à classificação das seis bandas TM com a Máxima Verossimilhança Gaussiana. Um estudo similar é apresentado em Braud e Feng (1998).

Estudos posteriores apresentaram variações como o uso da transformação tasseled cap (Huang et al. 2002 ou Scott et al., 2003), a transformação IHS (Zakariya et al. 2006) ou outros índices (Bhagat V.S. e Sonawane 2011). Também métodos de segmentação foram usados para delinear lagos, como mostrado em Di et al. (2003).

No Brasil, um dos primeiros estudos é encontrado em Carvalho et al. 1988, quem também aponta as vantagens do uso da banda do infra-vermelho próximo para esta finalidade. Um estudo mais recente, Silva et al. 2012, apresenta uma maneira de monitorar a evolução dos espelhos de água de um corpo de água, porém não se faz menção à acurácia obtida.

\section{3. ÁREA DE ESTUDO}

A Represa do Vossoroca localiza-se em Tijucas do Sul, na Região Metropolitana de Curitiba, Paraná. A represa está inserida na bacia do rio São João que é o principal rio, e possui os rios Vossoroca e Fojo além do São João como corpos formadores d'água.

Construída na década de 70, possui uma área de $3,3 \mathrm{~km}^{2}$ e volume de 35,7 milhões de $\mathrm{m}^{3}$, a profundidade média é de $8 \mathrm{~m}$ e a máxima é de $17 \mathrm{~m}$. Sua principal função é regular a vazão para a usina hidrelétrica de Chaminé, localizada a juzante, na margem esquerda do rio São João ( Blum et al., 2005).

A Usina de Chaminé foi construída pela Cia Força e Luz do Paraná, começando a operar em 1930. Possui capacidade instalada de $18 \mathrm{MW}$, está localizada na margem esquerda do Rio São João, a cerca de $80 \mathrm{~km}$ de Curitiba.

\section{MATERIAIS USADOS}

Neste estudo foram usadas imagens do sistema Landsat. As imagens utilizadas neste trabalho foram baixadas pela homepage da USGS (http://earthexplorer.usgs.gov/). Foram utilizadas imagens Landsat 8 e Lansat 7, órbita 220 e ponto 078.

2010 data 29/12/2010 sensor landsat 7;

2012 data 21/03/2012 sensor landsat 7;

2014 data $30 / 01 / 2014$ sensor landsat 8 ;

2015 data $01 / 01 / 2015$ sensor landsat 8 .

O satélite landsat 7 tem erros no varredor e por isso tem linhas sem informação na imagem (Fig. 1) e conseqüente ausência de dados dos sensor.

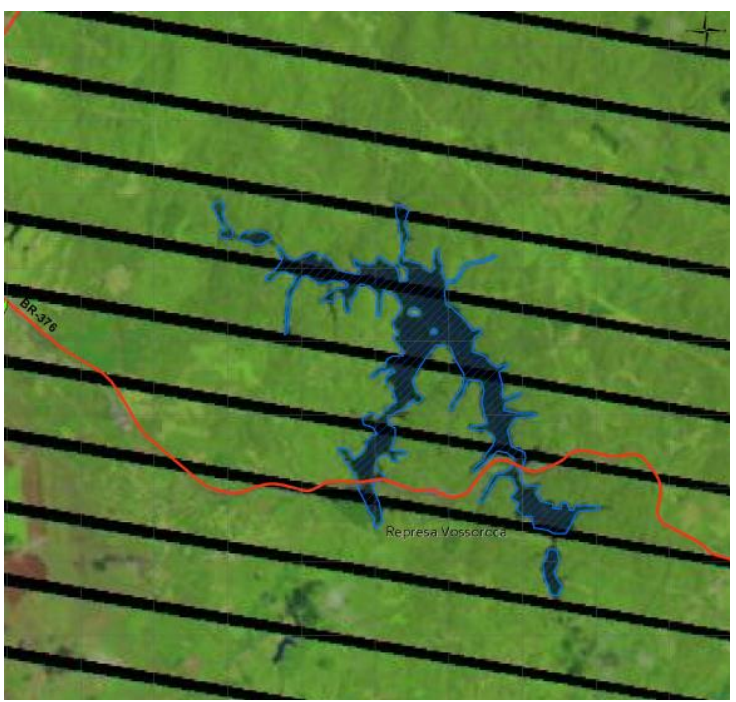

Figura 1 - Imagem Landsat ETM+ do verão 2010.

\section{METODOLOGIA}

Como primeiro passo foi realizado o georreferencimento das imagens. Por serem do mesmo sistema, um modelo simples, como o do polinômio de primeiro grau provou ser suficiente. Ainda, algumas imagens vieram já registradas, o que facilitou esta etapa.

A seguir procedeu-se a digitalizar os contornos do lago nas imagens. Com a delimitação de poligonais nas imagens de verão referentes aos anos de 2010, 2012, 2014 e 2015 foram calculadas estatísticas de área de cada data bem como foi feito o cruzamento destes polígonos para visualizar e avaliar o avanço da lâmina d'água e permitir um estudo comparativo entre 2010, 2012, 2014 e 2015.

No presente trabalho, para fazer a vetorização do espelho d'água e desenhar a poligonal, primeiro efetuouse a classificação supervisionada pelo método da Máxima Verossimilhança para se obter uma referência e o resultado da classificação foi otimizado por edição manual. Note-se que nas imagens Landsat 7 , devido à falha do sistema SLC existem áreas que não são imageadas, o que resulta em graves perdas para a classificação. A combinação destes dois métodos, automático e visual, mostrou ser eficiente, uma vez que permitiu fazer os ajustes necessários, através do 
detalhamento das características de cada imagem, interpretando visualmente cada poligonal do estudo.

A seguir, as mesmas imagens foram submetidas ao processo de segmentação pelo algoritmo Mean Shift, de (Comaniciu and Meer. 2002) disponível no software EDISON (Edge Detection and Image Segmentation) desenvolvido por Christoudias, Georgescu e Meer (Christoudias et al., 2002). O algoritmo assume que o espaço de cores pode ser modelado com uma função de densidade de probabilidade empírica. É assumido que se existem regiões uniformes na imagem, a representação dos pixels que formam uma região formaria um agrupamento, caracterizado por um máximo local, um pico da função densidade de probabilidade. Então ao detectar este pico se estaria achando a cor do segmento ou região uniforme.

Para detectar os picos, o algoritmo usa um processo iterativo. Considerando um pixel qualquer na imagem, uma região em torno do mesmo no espaço de cores é analisada. Se o pixel é a cor predominante no segmento, então ele seria o pico da região, o máximo local. Quando isto não ocorre, o processo se repete, deslocando o centro para o centróide da região. Com isto, a análise é direcionada a uma região mais densa dentro do espaço de cores. Este processo é repetido até detectar o pico. O valor da cor do pico é atribuído ao pixel inicial. Como vários pixels convergem para o mesmo pico, então o segmento é delineado na imagem simultaneamente. Detalhes do método podem ser encontrados em Lee et al (2010). Dois parâmetros devem ser estipulados pelo usuário: um parâmetro espacial, para definir o raio da vizinhança em torno do pixel e um parâmetro espectral que descreve a tolerância da diferença em termos de valor digital entre pixels.

Composições coloridas das imagens (infravermelho próximo, vermelho, verde) foram segmentadas usando o algoritmo Mean Shift. Porém, as imagens do Landsat 7 apresentam falhas, o que prejudica o desempenho do segmentador.

Em 2003, a NASA relatou uma falha no sistema de correção de varredura no ETM+, a bordo do Landsat 7. O mecanismo SLC, que tem a finalidade de compensar o movimento ao longo da linha de vôo e permite produzir linhas paralelas transversais, deixou de funcionar, com o que as imagens gravadas apresentam um padrão de "zigzag". Isto tem uma severa consequência, pois dados são perdidos nos extremos da imagem. Segundo Zhang et al. (2007) cerca de $22 \%$ da imagem total é perdida.

Com a finalidade compensar o efeito da falha do sistema ETM+, uma combinação multitemporal de bandas foi utilizada. Neste caso, as imagens do infravermelho próximo dos anos 2010, 2014 e 2015 foram usadas no processo de segmentação com o intuito de compensar a falta de pixels na imagem de 2010 .

\section{RESULTADOS}

Os produtos cartográficos resultantes da primeira fase da análise denotam a evolução ou recuo hídrico da represa, no intervalo de 5 anos.
A Fig. 1 apresenta o espelho de água do reservatório no ano de 2010. 2010 foi um ano chuvoso, sem os efeitos da estiagem, e por isso esta imagem foi adotada como referência para monitorar a evolução do espelho de água no período 2012-2015, como apresentado a seguir. A área do espelho de água em 2010 é de 361,621 ha. Neste caso, houve necessidade de edição manual devido à perda de linhas no sistema ETM+. Nos seguintes anos, os dados de imagens da mesma época do ano, verão, foram usados para avaliar a variação da reserva hídrica neste reservatório durante o período mais crítico.

A situação da reserva hídrica no reservatório do Vossoroca se agravou durante o verão de 2014, como pode ser visto na imagem apresentada na Fig. 2a. Em 2014, apesar de o inverno ter sido chuvoso, a represa está mais seca. A análise da imagem de satélite mostra que o espelho de água foi drasticamente reduzido. A digitalização das bordas do lago revelou que o espelho de água se reduziu a 159,317 hectares, conforme a Tab. 1 . Isto representa 44\% em relação à área verificada em 2010.

Em 2014, a presença de solo exposto nas margens da represa do Vossoroca aumentou, de acordo com a imagem de satélite Landsat 8 do dia 30/01/2014. Na região sudeste o solo exposto apresentou uma extensão de 37,0412 hectares, enquanto que na porção sudoeste é de 18,3078 hectares. Próximo da rodovia BR-376 a porção sudeste se destaca com uma área de 29,6522 hectares do lado direito, enquanto que na porção sudoeste, lado esquerdo, apresenta uma área de 12,7395 hectares.

No ano seguinte, durante o verão de 2015, o espelho de água cresceu em relação a 2014. A área atribuída ao reservatório é de 254,541 hectares, algo em torno de 70 \% da área de referência (verão de 2010).

Tabela 1 - Análise comparativa da área ao longo do tempo

\begin{tabular}{ccc}
\hline Ano & Lâmina d'água (ha) & $\%$ \\
\hline 2010 & 361,621 & 100 \\
2012 & 349,680 & 97 \\
2014 & 159,317 & 44 \\
2015 & 254,541 & 70 \\
\hline
\end{tabular}

A evolução da área do espelho de água entre 2010 e 2015 pode ser analisada com ajuda da Tab. 1. Nota-se que a área do espelho de água do lago atingiu um mínimo em 2014, o que significa que o volume do reservatório chegou ao mínimo neste ano. A situação melhorou drasticamente no verão de 2015, quando a área do lago chega a $70 \%$ do valor de referência, comprovando que a reserva hídrica na região aumentou devido às chuvas de 2014. 


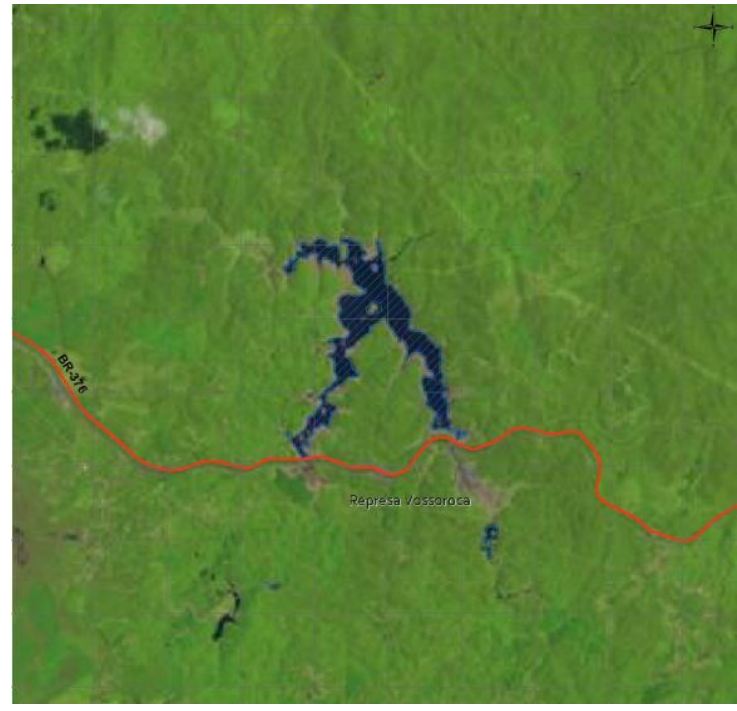

(a)

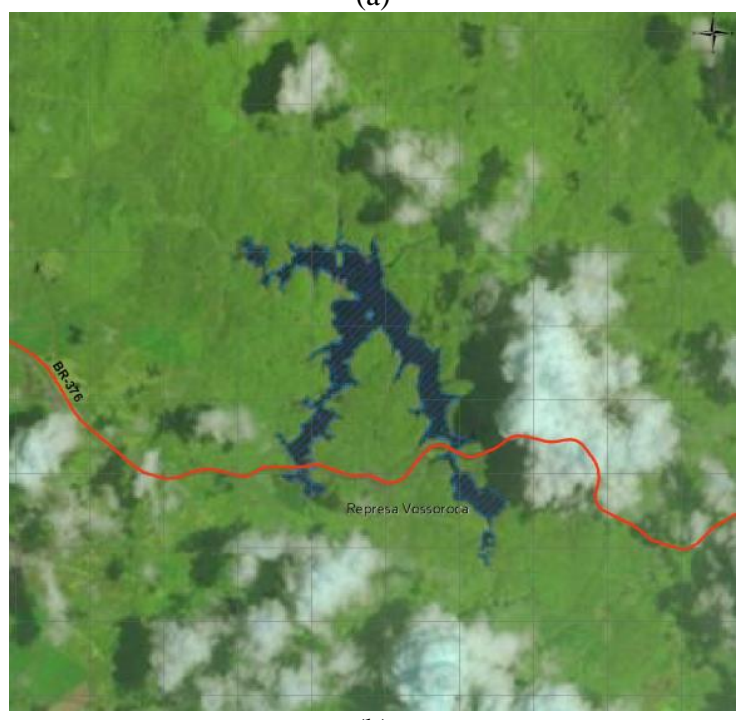

(b)

Figura 2 - Imagens Landsat 8 do verão 2014 e 2015.

Na segunda parte do estudo foi avaliada a possibilidade de reduzir a participação humana no processo através da segmentação da imagem. O segmentador Mean Shift foi aplicado a cada composição colorida. Os parâmetros foram definidos segundo a Tab. 2 .

Para fins de ilustração, o resultado da segmentação da imagem correspondente ao verão de 2014, maior estiagem, é mostrada na Fig. 3.

Comparando os valores obtidos pelos dois métodos é verificado que existe proporcionalidade entre os resultados, conforme pode ser visto na Fig. 4. Na mesma figura é incluída a linha tracejada que representa uma reta a 45 graus. Verifica-se que a segmentação subestima a área do reservatório em 2010 e 2015, enquanto que a superestima em 2015.
Tabela 2 - parâmetros da segmentação Mean Shift

\begin{tabular}{ccc}
\hline Ano & $\begin{array}{c}\text { Parâmetro } \\
\text { espacial }\end{array}$ & $\begin{array}{c}\text { Parâmetro de } \\
\text { cor }\end{array}$ \\
\hline 2010 & 30 & 5 \\
2012 & 10 & 8 \\
2014 & 2 & 8 \\
2015 & 20 & 3 \\
Multi(10/14/15) & 20 & 3 \\
\hline
\end{tabular}

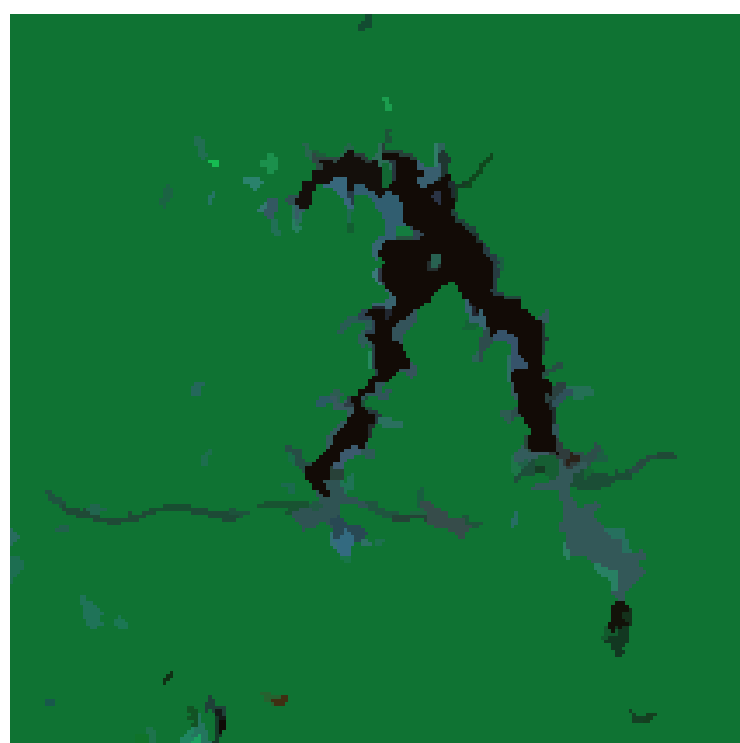

Figura 3 - Segmentação da imagem de 2014 com o algoritmo Mean Shift.

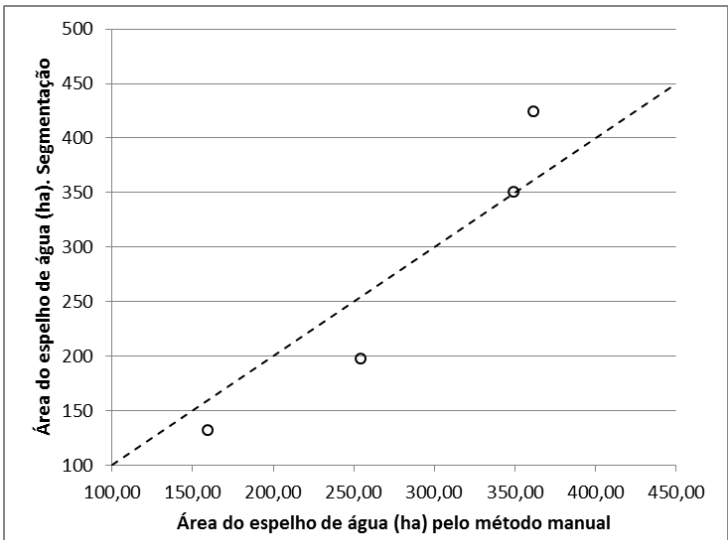

Figura 4 - Comparação da estimativa de área pelos dois métodos.

A análise foi repetida, desta vez considerando a variação relativa entre os dois experimentos. Os resultados são mostrados na Fig. 5. Nesta análise pode ser observado que existe grande similaridade entre os resultados. O método baseado na segmentação produz estimativas muito similares em termos de variação percentual do espelho de água em relação à área de 2010. Numericamente, as estimativas são sempre inferiores, variando entre $82 \%$ e $94 \%$. 


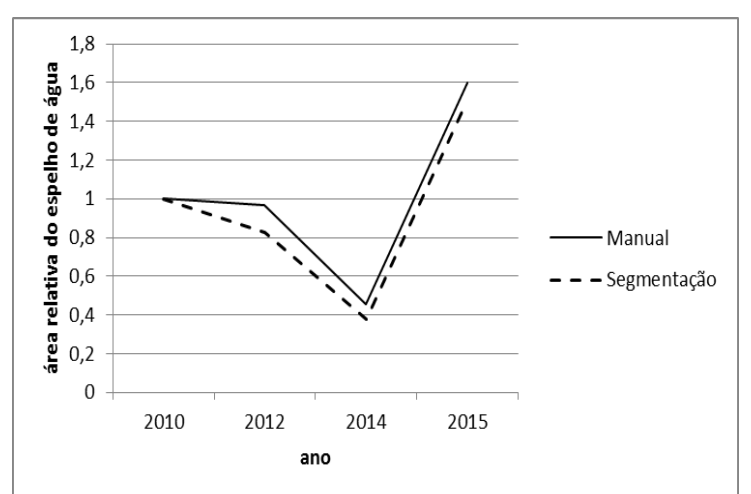

Figura 5 - Comparação da variação relativa do espelho de água usando como base 2010 .

\section{CONSIDERAÇÕES FINAIS}

Neste artigo se discutiu a variação do espelho d'água da represa do Vossoroca nos anos de 2010 a 2015 com base na análise de imagens do sistema Landsat. Estas imagens permitiram fazer comparações da área dos reservatórios num intervalo temporal e acompanhar um grave período de estiagem.

Através das imagens utilizadas, constatou-se que comparado com 2010, o volume da represa diminuiu consideravelmente em 2014, devido à estiagem. Em 2015, a represa já apresenta sinais de recuperação, apresentando um aumento no volume do reservatório.

O método baseado na segmentação Mean Shift produziu resultados diferentes do método manual, considerado aqui como referência. De forma geral, os resultados provam que pode ser aplicada a segmentação para acompanhar a variação relativa do espelho de água ao longo dos anos. Este método pode ser aplicado a mais de um reservatório para avaliar a disponibilidade hídrica de uma região.

\section{REFERÊNCIAS BIBLIOGRÁFICAS}

BHAGAT V.S.; SONAWANE K.R.. 2011. Use of LANDSAT ETM+ Data for delineation of water bodies in hilly zones. Journal of Hydroinformatics, 13(4), 661-671. DOI:10.2166/hydro.2010.018.

BLUM, C.T.; POSONSKI, M.; HOFFMANN, P.M.; BORGO, M.. 2005. Espécies vegetais invasoras em comunidades florestais nativas nas margens da Represa do Vossoroca, APA de Guaratuba, Paraná, Brasil. In: I Simpósio Brasileiro sobre Espécies Exóticas Invasoras, Brasília.

BRAUD; D.H.; FENG, W.. 1998. Semi-automated construction of the Luisiana coastline digital land/water boundary using Landsat Thematic Mapper satellite imagery. Technical Report 97-002, Department of Geography \& Anthropology, Luisina State University. Luisiana Applied Oil Spill Research and Development Program, OSRAPD.
NERY, J.T.; SILVA, E.S.. 2001. Análise da Precipitação no Estado do Paraná In: XI Congresso Argentino de Metereologia e IX Congresso FLISMET, Buenos Aires.

CARVALHO, G. M. B. de S.; SOARES, A. M. L.; ALMEIDA, M. A. G.; CRUZ, M. L.B.; MARTINS, M. L. R.; SOARES, Z. M. L.. 1988. Monitoramento dos espelhos d'água dos açudes do Estado do Ceará. Anais do $V$ Simpósio Brasileiro de Sensoriamento Remoto, Natal$R N$.

COMANICIU, D.; MEER, P.. 2002. Mean shift: a robust approach toward feature space analysis. Pattern Analysis and Machine Intelligence, IEEE Transactions on, Vol. 24, Nr. 5, may (2002), p. $603-619$.

Christoudias, C.; GeORgescu, B.; Meer, P.. 2002. Synergism in low-level vision. 16th International Conference on Pattern Recognition, Quebec City, Canada, vol. IV, 150-155.

DI K., WANG J.; MA R.; LI R.. 2003. Automatic shoreline extraction from high-resolution Ikonos satellite imagery. In: proceeding of the Annual ASPRS Conference. Anchorage, Alaska.

FRAZIER P.S.; PAGE, K. J.. 2000. Water body detection and delineation with landsat TM data. Photogrammetric Engineering and Remote Sensing, 66 (12): 1467-1467.

HUANG C., WYLIE B., HOMER C., YANG L. and ZYLSTRA G.. 2002. Derivation of a tasseled cap transformation based on Landsat 7 ETM at-satellite reflectance. International Journal of Remote Sensing, 23: 1741- 1748.

LEE, I-C.; CHENG, L.; LI., R. 2010. Optimal parameter determination for mean-shift segmentation-based shoreline extraction using lidar data, aerial orthophotos, and satellite imagery. Proceedings of the ASPRS 2010 Annual Conference, 26-30 April, San Diego, CA, 8 p. $(C D-R O M)$.

SCOTT, J.W.; MOORE, L.R.; HARRIS,W.M.; REED, M.D.. 2003. Using the Landsat 7 Enhanced Thematic Mapper Tasseled Cap Transformation to Extract Shoreline. Open-File Report OF 03-272 U.S. Geological Survey.

SILVA, C. R. ; LIMA, E. de P.; MACHADO, J. S. 2012. Análise temporal do espelho d'água da lagoa Parnaguá (PI) usando imagens digitais. Ambiência, Guarapuava, $P R$, v. 8, n. 3: 909 - 919.

ZAKARIYA R.; ROSNAN Y.; SAIDIN S.A.; YAHAYA M.H.; KASAWANI I.; LOKMAN H.. 2006. Shoreline detection and changes for Terengganu river mouth from satellite imagery (Landsat 5 and Landsat 7). Journal of Sustainability Science and Management, 1: 47-57. 
ZHANG, C.; LI, W.; TRAVIS, D. 2007.. Gaps-fill of SLC-off Landsat ETM+ satellite image using a geostatistical approach, International Journal of Remote Sensing, 28:22, $5103-5122$. 